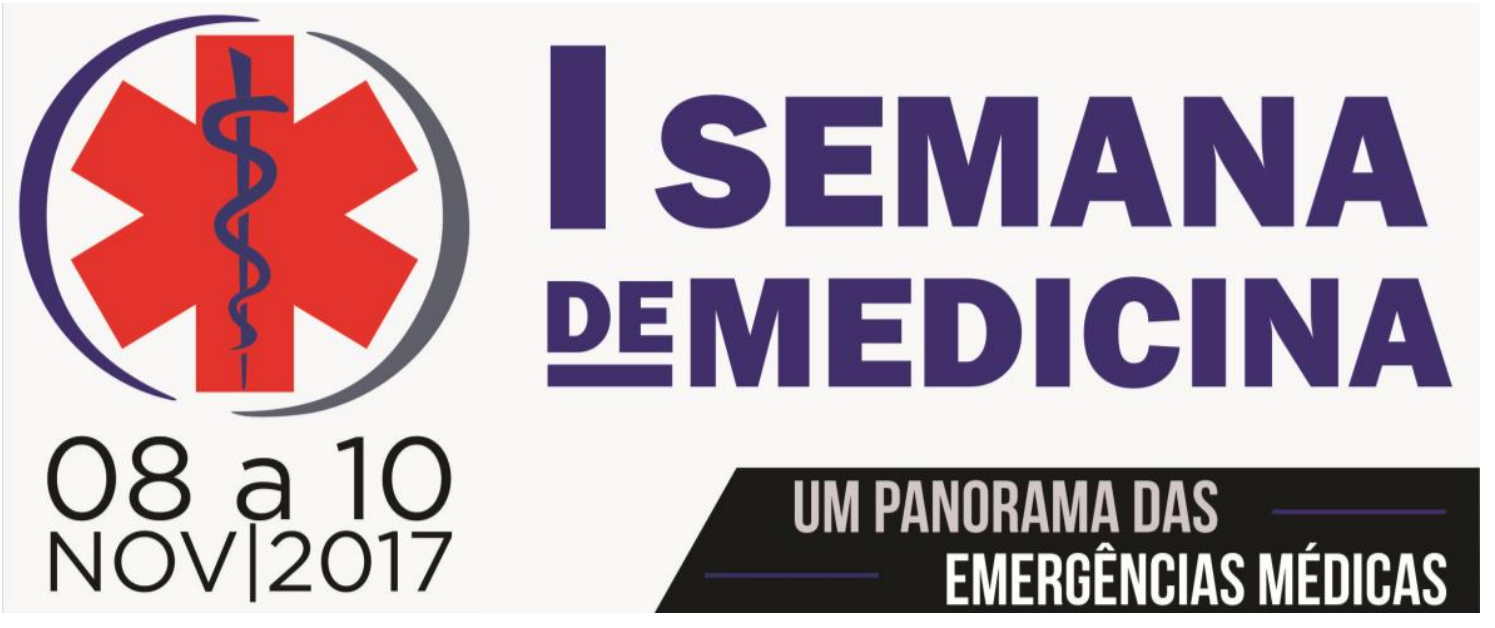

\title{
ANAIS DA I SEMANA DE MEDICINA DA UFCG: UM PANORAMA DAS EMERGÊNCIAS MÉDICAS
}

Realizada em 08 a 10 de novembro de 2017 


\title{
REITOR \\ Vicemário Simões
}

\section{VICE-REITOR}

Camilo Allyson Simões de Farias

PRÓ-REITOR DE PESQUISA E EXTENSÃO

Onireves Monteiro de Castro

\section{DIRETOR DO CENTRO DE FORMAÇÃO DE PROFESSORES}

Antônio Fernandes Filho

\author{
VICE-DIRETOR DO CENTRO DE FORMAÇÃO DE \\ PROFESSORES \\ Carlos Davdson Pinheiro
}

COORDENADOR ADMINISTRATIVO DA UNIDADE ACADÊMICA

DE CIÊNCIAS DA VIDA

Allan Pablo do Nascimento Lameira

COORDENADOR DO CURSO DE BACHARELADO EM MEDICINA Luiz Jardelino de Lacerda Neto

COORDENAÇÃO GERAL DA I SEMANA DE MEDICINA DA UFCG

Luiz Jardelino de Lacerda Neto

Jefferson Marlon de Medeiros Pereira Maciel

COMISSÃO CIENTÍFICA 
Allan Pablo do Nascimento Lameira André Barroso do Nascimento de Sousa Reis Andreza Guedes Barbosa Ramos

Antônio Humberto Pereira da Silva

Enágio Amorim Xavier

Flávio Lourenço de Oliveira

Francisco Geyson Fontenele Albuquerque

Geofábio Sucupira Casimiro

José Cesário de Almeirda

Maria do Carmo de Alustau Fernandes

Mariane Rodrigues Pires

Natália Bitu Pinto

Sávio Benvindo Ferreira

SECRETARIA GERAL

Paulo Soares de Andrade Filho

Luiza Carla de Medeiros Góis

Letícia Pinheiro de Melo

TESOURARIA

Ticiane Costa Farias

Maria Zilda Melo Regis

Hédulla Karoliny de Souza Lima Tavares

Evanildo Rodrigues de Sousa Junior

COMISSÃO DE INFRAESTRUTURA E LOGÍSTICA

Marlla Héllen do Nascimento Araújo

Karla Suellén de Araújo Souza

Lorena Alves de Souza Leal de Araújo 
Edilmax Araújo Marques dos Santos

Thaís Bernardino Lima

João Pedro Maciel Capistrano

COMISSÃO DE COMUNICAÇÃO

Anna Caroline Domingos Lima

Lucas Messias Augusto de Sousa

COMISSÃO DE PATROCÍNIO

Maria Stela Gomes Oliveira

Elias Figueiredo da Silva

Marina Cardoso Oliveira 


\section{RESUMOS}

FATORES PREDISPONENTES NA SEPSE NEONATAL TARDIA Nathalie Ramos Formiga Rolim ${ }^{1}$, Dulcy Dávyla Freire Nascimento ${ }^{1}$, Ana Maria Souza Costa Barros', Joyce Flávia da Silva Leal', Ana Emília Formiga Marques².

EFEITOS HEMATOLÓGICOS DA DIPIRONA: UMA REVISÃO BIBLIOGRÁFICA Rebeca Karollyne Rolim Ribeiro ${ }^{1}$, Lucas Messias Augusto De Sousa ${ }^{1}$, Rayllane Santos Nunes ${ }^{1}$, Natália Bitu Pinto ${ }^{2}$.

PERFIL EPIDEMIOLÓGICO DOS INTERNAMENTOS POR INSUFICIÊNCIA CARDÍACA NA REGIÃO NORDESTE DO BRASIL: SÉRIE TEMPORAL DE 2010 A 2014 Leandro Januário de Lima ${ }^{1}$, Mateus de Oliveira Medeiros ${ }^{1}$, Maria do Carmo Andrade Duarte de Farias ${ }^{2}$.

LIPOFUSCINOSE CERÓIDE NEURONAL: RELATO DE CASO Wenya Cristiana de Almeida Abreu, Dieglys de Santana Sarmento ${ }^{1}$, Francisco Anderson de Sá Carvalho ${ }^{1}$, Luciano Mota Reis Neto ${ }^{1}$, Luciana Modesto de Brito $^{2}$.

REVISÃO SISTEMÁTICA: USO DA PREGABALINA NO TRATAMENTO DA DOR NEUROPÁTICA Marília Millena Remígio da Costa ${ }^{1}$, Edilberto Souza ${ }^{1}$, David Henrique Vieira Vilaça ${ }^{1}$, Natália Bitu Pinto ${ }^{2}$.

RELATO DE EXPERIÊNCIA: CONTRIBUIÇÃO DA MONITORIA DE SEMIOLOGIA MÉDICA PARA APERFEICOAMENTO DO ENSINO APRENDIZAGEM Marília Millena Remígio da Costa', Antônio José Barbosa Neto ${ }^{1}$, Paulo Antônio Farias Lucena ${ }^{2}$.

AVALIAÇÃO DO EFEITO DA METFORMINA NA SÍNDROME DO OVÁRIO POLICÍsTICO Anna Caroline Domingos Lima ${ }^{1}$, Fernanda Maria Gomes Carvalho ${ }^{1}$, Maria Zilda Melo Regis ${ }^{1}$, Natália Bitú Pinto ${ }^{2}$.

TAXA DE DETECÇÃO DE ACIDENTES POR ANIMAIS PEÇONHENTOS Maria Zilda Melo Regis ${ }^{1}$, Ticiane Costa Farias ${ }^{1}$, Hédulla Karoliny De Souza Lima Tavares ${ }^{1}$, Márlla Héllen Do Nascimento Araújo ${ }^{1}$, Paula Frassinetti Oliveira Cezário ${ }^{2}$.

A IMPORTÂNCIA DO CONHECIMENTO DA BIOMECÂNICA DO TRAUMA NOS FERIMENTOS POR ARMA DE FOGO Luciano Mota Reis Neto ${ }^{1}$, David Sammuel Dantas Torres ${ }^{1}$, Yolanda de Melo Omena Lira ${ }^{1}$, Wenya Cristiana de Almeida Abreu', Luciana Modesto de Brito ${ }^{2}$.

SíFILIS CONGÊNITA: UM DESAFIO PARA A SAÚDE PÚBLICA Rayllane Santos Nunes ${ }^{1}$, Marlla Héllen do Nascimento Araújo ${ }^{1}$, Marcos Alan de Sousa Barbosa $^{1}$, Rebeca Karollyne Rolim Ribeiro ${ }^{1}$, Rayanne de Sousa Barbosa ${ }^{2}$.

RECRUTAMENTO PULMONAR COM PRESSÃO POSITIVA EXPIRATÓRIA FINAL (PEEP) NA SÍNDROME DO DESCONFORTO RESPIRATÓRIO AGUDO (SDRA): UMA REVISÃO BIBLIOGRÁFICA Francisco Jaime de 
Araujo Filho ${ }^{1}$, Lucas Gomes de Freitas Lima ${ }^{1}$, Samylly Teixeira de Araújo ${ }^{1}$, Leonardo Pereira Tavares ${ }^{1}$, José Benício Dantas Neto².

PERFIL EPIDEMIOLÓGICO DA HANSENÍASE EM UMA CIDADE DA MESORREGIÃO DO SERTÃO PARAIBANO: UM ESTUDO ECOLÓGICO Marcos Alan Sousa Barbosa', Ana Paula Barbosa Nóbrega', João Pedro Maciel Capistrano ${ }^{1}$, Rayllane Santos Nunes ${ }^{1}$, Natália Bitu Pinto ${ }^{2}$.

CONDUTAS DE URGÊNCIA UTILIZADAS EM FRATURAS DO ANEL PÉLVICO Edilberto Costa Souza ${ }^{1}$, Marília Millena Remígio da Costa ${ }^{1}$, Vanessa Lima Gonçalves ${ }^{1}$, Francisco Anderson de Sá Carvalho', Sheylla Nadjane Batista Lacerda ${ }^{2}$.

POUCA ADESÃO POPULACIONAL AOS PROGRAMAS DE SAÚdE DA ATENÇÃO BÁSICA: UM RELATO DE EXPERIÊNCIA NO BAIRRO ASA DO MUNIĆ́PIO DE CAJAZEIRAS-PB Maíra Pacheco Fraga ${ }^{1}$, Leandro Carvalho de Souza ${ }^{1}$, Leandro Santana Ferreira ${ }^{1}$, Wengna Neves Matias ${ }^{1}$, Ocilma Barros de Quental ${ }^{2}$.

MONITORIZAÇÃO HEMODINÂMICA INVASIVA Vanessa Lima Gonçalves', Edilberto Costa Souza ${ }^{1}$, Leonardo Victor de Moraes Galdino ${ }^{1}$, Alana Cristina Alves Garcia ${ }^{1}$, Sheylla Nadjane Batista Lacerda ${ }^{2}$.

FATORES QUE INTERFEREM NA HIPERBILIRRUBINEMIA NEONATAL: UMA REVISÃO DE LITERATURA Dulcy Dávyla Freire Nascimento', Ana Maria Souza Costa Barros ${ }^{1}$, Nathalie Ramos Formiga Rolim¹, Joyce Flávia da Silva Leal', Ana Emília Formiga Marques². 


\title{
FATORES PREDISPONENTES NA SEPSE NEONATAL TARDIA
}

\author{
Nathalie Ramos Formiga Rolim ${ }^{1}$, Dulcy Dávyla Freire Nascimento ${ }^{1}$, Ana Maria \\ Souza Costa Barros ${ }^{1}$, Joyce Flávia da Silva Leal ${ }^{1}$, Ana Emília Formiga \\ Marques $^{2}$. \\ ${ }^{1}$ Faculdade Santa Maria (FSM). Email: nathalieramosf@hotmail.com \\ ${ }^{2}$ Faculdade de Medicina de Juazeiro do Norte (FMJ). Email: \\ anaemiliaformiga@hotmail.com
}

\begin{abstract}
RESUMO
INTRODUÇÃO: Mesmo diante do avanço dos cuidados neonatal, A sepse neonatal é uma das principais causas de morte dos recém-nascidos (RN) em todo mundo e configura-se como um dos fatores que mais contribui para a elevação do índice de mortalidade neonatal. OBJETIVOS: Apresentar uma revisão de literatura a respeito dos fatores predisponentes de sepse neonatal tardia. MATEIRAIS E MÉTODOS: Realizou-se uma busca nos bancos de dados MEDLINE, LILACS e SciELO de artigos que tratem da sepse neonatal que houvessem sido publicados de 2005 a 2017. Para o levantamento foram utilizados os descritores: sepse neonatal, sepse neonatal tardia e mortalidade neonatal. RESULTADOS E DISCUSSÕES: Os autores destacam que os fatores predisponentes estão relacionados com as condições próprias do $\mathrm{RN}$ e com os procedimentos realizados no ambiente hospitalar. Os $\mathrm{RN}$ de baixo peso, os prematuros extremos e os submetidos a procedimentos invasivos nas unidades de terapia intensiva. Os cateteres venosos centrais são os maiores fatores de risco para a infecção corrente sanguínea em uma UTI neonatal. Intubações repetidas, uso de drenos, permanência em ventilação mecânica prolongada, uso de nutrição parenteral, uso de antibiótico de amplo espectro, superlotação das unidades de internação com número de pessoal insuficiente na assistência direta ao RN e tempo de hospitalização prolongado tem sido relatado como fatores de risco de infecção tardia hospitalar. CONCLUSÕES: A Sepse neonatal é uma doença grave que precisa ser diagnosticada precocemente para o tratamento adequado, evitando assim a chance de evolução a óbito, para isso é importante uma maior atenção em crianças que nascem diante dos fatores discutidos.
\end{abstract}

Palavras-chaves: Sepse neonatal; Fatores de Risco; Neonatologia.

RPI Revista de Pesquisa Interdisciplinar, Cajazeiras, v. 2, Ed. Especial, 1 - 22 nov. 2017. 


\title{
EFEITOS HEMATOLÓGICOS DA DIPIRONA: UMA REVISÃO BIBLIOGRÁFICA
}

\author{
Rebeca Karollyne Rolim Ribeiro ${ }^{1}$, Lucas Messias Augusto De Sousa ${ }^{1}$, Rayllane Santos \\ Nunes ${ }^{1}$, Natália Bitu Pinto ${ }^{2}$. \\ ${ }^{1}$ Universidade Federal de Campina Grande (UFCG). Email: rebeca.rrg@hotmail.com \\ ${ }^{2}$ Universidade Federal de Campina Grande (UFCG).Email: nataliabitu@gmail.com
}

\begin{abstract}
RESUMO
INTRODUÇÃO: A dipirona é, notoriamente, um fármaco que apresenta ampla margem de prescrição. Este medicamento tem como principais efeitos o analgésico e o antitérmico. No entanto, há suspensão da sua comercialização em alguns países como Estados Unidos, Reino Unido e Noruega devido à incidência de agranulocitose e às divergências no que tange ao seu uso racional, sua respectiva segurança e efeitos adversos. Por outro lado, no Brasil o consumo da dipirona é concedido sem restrições, revelando o status de principal analgésico da terapêutica brasileira. Apesar disso, poucos estudos e casos têm abordado as alterações hematológicas deste fármaco. OBJETIVOS: Nesse raciocínio, o objetivo deste estudo é propor um resgate das discussões desenvolvidas relativas aos efeitos hematológicos fomentados por meio da dipirona, ressaltando a confiabilidade e qualidade do seu uso. MATERIAIS E METODOLOGIA: Realizou-se uma busca nos bancos de dados MEDLINE, LILACS e SciELO de artigos que tratem dos efeitos hematológicos da dipirona que houvessem sido publicados de 1985 a 2010. Para o levantamento foram utilizados os descritores: dipirona, agranulocitose e discrasias sanguíneas. RESULTADOS E DISCUSSÕES: Considerando que o emprego desse medicamento está relacionado sobretudo ao seu caráter de indicação analgésico e antipirético, relata-se sua imensa difusão pela população mundial. Aliado a isso, vale citar que as condições de baixo custo, ampla disponibilidade e isenção de prescrição corroboram também com 0 processo de automedicação. Por outro lado, estudos realizados nos âmbitos eletrofisiológicos, comportamentais e neuroquímicos indicam que o mecanismo de ação desse analgésico é ainda controverso. Isso se dá, pois a ação da dipirona ocorre em nível do sistema nervoso central e periférico, assim como em outros níveis de processamento da informação referente à dor. Por conseguinte, no que tange à contra-indicação, levantamentos bibliográficos revelam que sua ação pode desencadear reações adversas graves, colocando em xeque a segurança do uso de tal fármaco. Sendo assim, em uma cadeia de efeitos adversos, aquele que recebe maior destaque é a supressão da formação de glóbulos brancos, em particular os granulócitos (agranulocitose), descrito em 1934. Os debates acerca de tal efeito colateral são suscitados principalmente pela razão de que os sintomas e os sinais da agranulocitose - como febre, tremores, amigdalite e dor de cabeça - serem os mesmos que induzem ao uso de analgésicos e antipiréticos. Além disso, determinados fatores genéticos estão sendo analisados na predisposicão do paciente às alterações hematológicas por dipirona. CONCLUSÃO: Infere-se, portanto, que a relação entre a dipirona sódica com alguns acometimentos hematológicos evidencia relevância significativa no âmbito do consumo farmacêutico nas emergências médicas no Brasil. Posto isso, faz-se necessário uma especial atenção em setores de emergência e de ambulatório às interações medicamentosas que induzam efeitos hematológicos indesejáveis. Tal fator confere relevância perante uma ação preventiva de repensar a real segurança e uso da dipirona para uma reversão de quadros clínicos graves.
\end{abstract}

Palavras-chave: Agranulocitose; Dipirona; Efeitos Hematológicos

RPI Revista de Pesquisa Interdisciplinar, Cajazeiras, v. 2, Ed. Especial, 1 - 22 nov. 2017. 


\title{
PERFIL EPIDEMIOLÓGICO DOS INTERNAMENTOS POR INSUFICIÊNCIA CARDÍACA NA REGIÃO NORDESTE DO BRASIL: SÉRIE TEMPORAL DE 2010 A 2014
}

\author{
Leandro Januário de Lima ${ }^{1}$, Mateus de Oliveira Medeiros ${ }^{1}$, Maria do Carmo \\ Andrade Duarte de Farias ${ }^{2}$. \\ ${ }^{1}$ Universidade Federal de Campina Grande (UFCG). Email: \\ leandrojanuario100@gmail.com \\ ${ }^{2}$ Universidade Federal de Campina Grande (UFCG). Email: \\ carmofarias0@gmail.com
}

INTRODUÇÃO: A insuficiência cardíaca (IC) é a via final de várias doenças que acometem o coração, e no contexto atual vem se consolidando como uma doença muito prevalente, em virtude de fatores como o envelhecimento da população. OBJETIVO: Compreender a série temporal das hospitalizações por IC e suas relações com as variáveis sociodemográficas. MÉTODOS: Pesquisa descritiva quanto aos seus objetivos, de cunho quantitativo, constituindose como uma pesquisa epidemiológica retrospectiva de série temporal, do tipo Ecológico. Utilizaram-se nesta análise os dados secundários disponíveis no Sistema de Informações Hospitalares do Sistema Único de Saúde (SIH/SUS). RESULTADOS: Na série temporal em questão, a Região Nordeste passou de 63.647 internamentos em 2010 para 54.178 em 2014, uma redução percentual de cerca de $14,9 \%$ em cinco anos. Verificou-se que maioria dos internamentos é realizada sob o regime de urgência no atendimento, além de que o setor público é o responsável pela maioria das hospitalizações contando com grande participação do setor privado. A população mais hospitalizada por insuficiência cardíaca (IC) é aquela acima de 50 anos, com destaque aqueles que possuem entre 70 e 79 anos, que mostrou a maior prevalência em todos os anos da série temporal. Em ambos os sexos a prevalência vem caindo, com o sexo masculino concentrando durante toda a série a maior quantidade de internamentos anualmente. Contudo, a diferença no número de hospitalizações apresenta-se em queda, criando-se uma tendência que nos próximos anos possa haver um empate neste quesito. CONCLUSÃO: O perfil nordestino contrasta com a tendência brasileira de aumento da prevalência da insuficiência cardíaca. Assim, como este estudo abordou os internamentos por esta causa, fatores como a melhora nas técnicas de tratamento podem corroborar para a redução das hospitalizações sem, contudo, alterar a prevalência da morbidade.

Palavras-chave: Insuficiência Cardíaca; Hospitalização; Pesquisa sobre Serviços de Saúde. 


\title{
LIPOFUSCINOSE CERÓIDE NEURONAL: RELATO DE CASO
}

\author{
Wenya Cristiana de Almeida Abreu ${ }^{1}$, Dieglys de Santana Sarmento ${ }^{1}$, Francisco \\ Anderson de Sá Carvalho ${ }^{1}$, Luciano Mota Reis Neto ${ }^{1}$, Luciana Modesto de \\ Brito ${ }^{2}$. \\ ${ }^{1}$ Faculdade Santa Maria (FSM). Email: wenyaabreu@hotmail.com \\ ${ }^{2}$ Faculdade Santa Maria (FSM). Email: lucianamodesto@hotmail.com
}

\begin{abstract}
RESUMO
INTRODUÇÃO: Lipofuscinose ceróide neuronal (LCN) constitui um grupo de doenças neurodegenerativas caracterizadas pelo depósito anormal de uma substância autofluorescente de lipopigmentos, que lembra ceróide e lipofuscina, dentro dos lisossomos dos neurônios e outros tipos de células. Sendo uma doença de transmissão autossômica recessiva que ocorre devido a uma mutação no gene CNL3; Este gene codifica uma proteína cuja função permanece desconhecida até ao momento. Caracterizada clinicamente por deterioração cognitiva, epilepsia mioclônica (com crises epilépticas que se caracterizam por contrações súbitas de grupamentos musculares, levando a deslocamento de um segmento corporal ou do tronco), perda visual progressiva, sintomas extrapiramidais, alterações hormonais especialmente em meninas, além de distúrbios psiquiátricos e do sono. OBEJETIVO: Avaliar as hipóteses fisiopatológicas possivelmente subjacentes à LCN, as opções terapêuticas atualmente disponíveis para o tratamento e as perspectivas futuras de investigação nesta área. METODOLOGIA: Realizou-se uma revisão integrativa qualitativa observacional através da revisão de prontuário como também a execução da anamnese completa da paciente, juntamente com seu exame físico. RESULTADOS E DISCUSSÕES: FPB, 13 anos, é a primeira filha de um casal jovem, saudável consanguíneo. Nasceu de parto normal, sem complicações, com peso de $2750 \mathrm{~g}$, com $48 \mathrm{~cm}$ e perímetro cefálico de $37 \mathrm{~cm}$. Seu desenvolvimento neuropsicomotor teve como marcos sentar sem apoio aos 8 meses, andar sem apoio com 1 ano e 4 meses e iniciou palavras perto dos 1 ano e 8 meses. Até os 4 anos conseguia correr, brincar e comer sozinha. Próximo aos 5 anos iniciou com frequentes crises convulsivas mioclônicas, com involução do seu desenvolvimento. Foi observada perda motora, involução na fala, perda na capacidade de controle esfincteriano e perda da marcha autônoma. Tomografia computadorizada realizada em novembro de 2008 demonstrava atrofia cerebral difusa. No início de 2011 foi encaminhada para avaliação com neuropediatra em Fortaleza no Hospital SARAH, que concluiu o diagnóstico de LCN. A menor atualmente alimenta-se por gastrostomia, apresenta perda visual, afagia, anorexia seguida de frequentes internações no HUJB da cidade de Cajazeiras por quadros recorrentes de pneumonia, infecção urinaria e lesão por pressão em região sacral. CONCLUSÃO: A evolução desta patologia foi acompanhada na paciente descrita acima e, de fato, corrobora as descrições da literatura. Outro aspecto que fica evidente é o caráter degenerativo da doença, visto que a paciente estudada teve seu quadro clínico deteriorado gradativamente, perdendo suas funções motoras e cognitivas paulatinamente com a evolução da doença. A taxa de sobrevida é baixa e o tratamento é baseado nas complicações existentes tendo em vista a necessidade de uma equipe multidisciplinar tanto para a menor quanto para os familiares.
\end{abstract}

Palavras-chave: Lipofuscinose ceróide neuronal; Epilepsia; Lisossomos. 


\title{
REVISÃO SISTEMÁTICA: USO DA PREGABALINA NO TRATAMENTO DA DOR NEUROPÁTICA
}

\author{
Marília Millena Remígio da Costa ${ }^{1}$, Edilberto Souza ${ }^{1}$, David Henrique Vieira \\ Vilaça ${ }^{1}$, Natália Bitu Pinto ${ }^{2}$. \\ ${ }^{1}$ Faculdade Santa Maria (FSM).Email: mariliamillena@gmail.com \\ ${ }^{2}$ Universidade Federal Campina Grande(UFCG). Email: nataliabitu@gmail.com
}

\begin{abstract}
RESUMO
INTRODUÇÃO: A neuropatia diabética dolorosa afeta $10 \%$ a $26 \%$ dos pacientes com diabetes. A dor neuropática, é causada por uma lesão ou doença que afeta o sistema nervoso somatossensorial, tem um impacto considerável na qualidade de vida dos pacientes, e está associada a uma elevada carga econômica para 0 indivíduo e a sociedade (HAANPÄÄ,2014). O tratamento com a pregabalina mostrou-se eficaz na redução de alguns tipos de dor neuropática, incluindo neuralgia e neuropatia periférica diabética (FINNERUP et al ,2015). OBJETIVO: O presente estudo teve como objetivo realizar uma revisão sistemática acerca do uso da pregabalina no tratamento da dor neuropática. METODOLOGIA: Nesta revisão incluímos estudos clínicos prospectivos e randomizados em humanos que mostraram a eficácia do uso da pregabalina em relação a outros fármacos, em pacientes com dor neuropática, com placebo ou nenhum tratamento para o controle da dor. Fizemos uma busca eletrônica nas bases de dados PubMed, PubMed Central, Embase e Central com as seguintes palavras-chave, Pregabalina; Neurite Plexo Braquial; Tratamento Farmacológico, para descobrir os estudos clínicos elegíveis em janeiro a setembro de 2017. Para a seleção do estudo, utilizamos estudos clínicos prospectivos e randômicos com humanos, que compararam uso da pregabalina com placebo ou nenhum tratamento da dor neuropática. Não impusemos restrição a qualquer idioma. Estudos de população adulta ou pediátrica foram incluídos, assim como, não fizemos busca por estudos não publicados, também não foram solicitados aos autores dados não publicados nos estudos incluídos. Como critérios de exclusão, foram estudos clínicos nos quais a pregabalina e outros fármacos foram comparados com placebo em populações que não tinha dor neuropática, estudos que não relataram os efeitos do fármaco do estudo sobre dor neuropática. Os resumos de estudos potencialmente elegíveis foram pesquisados manualmente para determinar a sua elegibilidade nesta revisão. Coletamos os dados necessários a partir do texto completo dos estudos. Inicialmente, todos os dados foram tabulados na planilha de cálculo do Microsoft Excel. Os seguintes dados foram extraídos dos estudos elegíveis: nome do primeiro autor, ano de publicação, métodos de randomização, população do estudo, protocolo de administração do fármaco no estudo, efeitos adversos da droga. RESULTADOS E DISCUSSÃO: A pesquisa nos bancos de dados revelou 33 artigos. Em 22 estudos clínicos, a pregabalina foi utilizada no tratamento da dor neuropática, de forma isolada mostrando eficácia, boa tolerabilidade e poucos efeitos adversos em $75 \%$ dos pacientes comparados a outros fármacos, em 10 estudos clínicos a pregabalina se mostrou eficaz mais associados gabapentina, porém apresentou muitos efeitos adversos principalmente na população idosa, em 1 estudo uso da pregabalina mostrou-se pouco eficiente quando associados as outras drogas. CONCLUSÃO: A gravidade da dor e a presença de outras comorbidades comumente resulta em uma situação em que os pacientes recebem múltiplas tratamentos simultaneamente. No entanto, algumas dificuldades estão associadas com essa abordagem, porém, a pregabalina mostrou-se eficaz, com a boa aceitação dos paciente
\end{abstract} quanto aos efeitos colaterais e com baixa interação medicamentosa.

Palavras-chave: Pregabalina; Neurite Plexo Braquial; Tratamento Farmacológico. 


\title{
RELATO DE EXPERIÊNCIA: CONTRIBUIÇÃO DA MONITORIA DE SEMIOLOGIA MÉDICA PARA APERFEICOAMENTO DO ENSINO APRENDIZAGEM
}

\author{
Marília Millena Remígio da Costa ${ }^{1}$, Antônio José Barbosa Neto ${ }^{1}$, Paulo Antônio \\ Farias Lucena ${ }^{2}$. \\ ${ }^{1}$ Faculdade Santa Maria (FSM).Email:.mariliamillena@gmail.com \\ ${ }^{2}$ Faculdade Santa Maria (FSM). Email: pauloflucena@yahoo.com.br
}

\begin{abstract}
RESUMO
INTRODUÇÃO: A disciplina de Semiologia Médica, consiste na introdução à fase clínica da graduação, trata-se de uma disciplina eminentemente prática e representa o primeiro momento do curso em que os alunos passam a ter sistematicamente contato com pacientes, objetivando ensinar técnicas de anamnese e exame físico, competências essenciais para 0 desenvolvimento de um raciocínio clínico acurado, havendo, portanto, menor dependência de exames complementares, fatos relevantes para todo médico generalista (MANGIORE, 2001, p. 210). OBJETIVO: Descrever, através de relato de experiência, a nossa vivência como monitores voluntários da disciplina de Semiologia Médica do aparelho respiratório, com alunos do quarto período do curso de Medicina da FSM, do período de fevereiro à outubro de 2017. MÉTODO: Trata-se de um estudo do tipo relato de experiência, da prática de monitoria voluntária da disciplina de Semiologia Médica do aparelho respiratório, é um componente curricular obrigatório do quarto período do curso de Medicina. Apresenta caráter teórico-prático, com carga horária de 10 horas semanais, sendo ministrada por dois monitores, no período de fevereiro à outubro de 2017. Inicialmente os discentes são submetidos a todo o conteúdo proposto na ementa, através de aulas teóricas, foram utilizados vídeo-aulas sobre os temas, treinamento das habilidades semiológicas (anamnese e exame físico do aparelho respiratório), com manequim disponibilizado no laboratório de habilidades, discussão de casos clínicos e realização de simulado para treinamento para prova prática. RESULTADOS E DISCUSSÃO: A monitoria é uma ferramenta benéfica que traz contribuições para a tríade: Professor-orientador, aluno-monitor e discente. $O$ primeiro terá um auxílio para a transmissão do conhecimento. $O$ aluno monitor é o estudante que se aproxima de área de conhecimento e junto a ela, realiza pequenas tarefas ou trabalhos, que contribuem para o ensino dos estudantes. Durante a disciplina de semiologia médica do aparelho respiratório as atividades realizadas pelo monitor vão desde participação na preparação de material didático, orientação e esclarecimento de dúvidas dos alunos, organização dos materiais do laboratório e acompanhamento das aulas teóricas/práticas bem como das avaliações. Como monitores, destacamos a importância dessas atividades para o desenvolvimento das mais diversas habilidades. Precisávamos rever os conteúdos aprendidos tentando desenvolver uma forma de repassá-los de forma didática. Além disso, era necessário dominar as técnicas de manuseio dos materiais bem como aprender a lidar com as angústias e frustrações de alguns estudantes. Dessa forma, também surgiu em nós uma atenção a mais para a docência. CONCLUSÃO: Diante do exposto, a monitoria tinha suas atividades planejadas para conseguir atender as demandadas dos alunos durante todas as atividades a serem desenvolvidas, desde a teoria à prática. Os ensinamentos adquiridos nessa experiência nos permitiu fortalecer o ensino aprendizagem, revelando-nos novas perspectivas acadêmicas.
\end{abstract}

Palavras-chave: Educação Médica; Monitoria; Docente; Discente. 


\title{
AVALIAÇÃO DO EFEITO DA METFORMINA NA SÍNDROME DO OVÁRIO POLICÍSTICO
}

\author{
Anna Caroline Domingos Lima ${ }^{1}$, Fernanda Maria Gomes Carvalho ${ }^{1}$, Maria Zilda \\ Melo Regis ${ }^{1}$, Natália Bitú Pinto ${ }^{2}$. \\ ${ }^{1}$ Universidade Federal de Campina Grande (UFCG). Email: \\ annacarolinedomingoslima@gmail.com \\ ${ }^{2}$ Universidade Federal de Campina Grande (UFCG). Email: \\ nataliabitu@gmail.com
}

\begin{abstract}
RESUMO
A síndrome dos ovários policísticos (SOP) é a endocrinopatia mais comum na fase reprodutiva da mulher; com prevalência entre 4 e 12\%. A ocorrência familiar é freqüente, principalmente quando há parentesco de primeiro grau. O conceito da SOP é muito amplo; caracteriza-se clinicamente pela presença de disfunção menstrual, hiperandrogenismo e anovulação crônica. Recentemente, drogas sensibilizadoras à insulina têm sido recomendadas como alternativa terapêutica de longo prazo no tratamento da SOP. Diante disso, essa pesquisa tem como objetivo avaliar a utilização da metformina em pacientes com síndrome do óvario policístico (SOP) e assim esclarecer e elucidar informações sobre tal medicamento em pacientes com tal síndrome através de uma revisão bibliográfica. Realizou-se uma busca nos bancos de dados MEDLINE, LILACS e SciELO de 2005 a 2017. Para o levantamento foram utilizados os descritores: "Síndrome dos Ovários Policísticos"; "Metformina"; "Hiperandrogenismo". Não houve restrição quanto à data de publicação dos artigos e idioma. A busca de artigos resultou na seleção de 8 artigos, foram excluídos estudos de revisão, estudos de caso, capítulos de livro, editoriais. Foi observado que a metformina se mostrou mais eficaz em reduzir a hiperandrogenemia. O hiperandrogenismo é uma das três principais características da SOP e tem como principal marcador o hirsurtismo, ou seja, o que foi possível concluir com o estudo é que o uso de metformina em pacientes com SOP diminuiu o aparecimento anormal de pêlos, uma das características negativas que mais incomodavam os pacientes com tal condição. Foi possível observar também que com o passar dos anos, mulheres portadoras da SOP, acabam por desenvolver uma resistência insulínica, a qual juntamente com a hiperinsulinemia caracteriza-se pela diminuição da sensibilidade dos tecidos à ação a insulina. E a metformina (biguanida) é um agente antidiabético de uso oral, derivado da guanidina, que atua reduzindo a hiperglicemia através do aumento da sensibilidade periférica a insulina o que promove a diminuição dos níveis glicêmicos. O uso de metformina diminui o peso corporal, devido a seu efeito anorexígeno, sendo benéfico para diminuição das taxas de triglicerídeos e colesterol. Também tem efeito protetor sobre os fatores de risco de angiopatia. Em pacientes não diabéticos, a metformina não estimula a secreção de insulina, não tendo, por isso, ação hipoglicemiante. Ela não provoca hipoglicemia e não causa hiperinsulinemia. Portanto podemos concluir com a análise de tais informações que a metformina é um medicamento que atua positivamente em pacientes com SOP, diminuindo consideravelmente alguns sinais característicos de tal condição.
\end{abstract}

Palavras-chave: Síndrome dos Ovários Policísticos; Metformina; Hiperandrogenismo. 


\title{
TAXA DE DETECÇÃO DE ACIDENTES POR ANIMAIS PEÇONHENTOS
}

\author{
Maria Zilda Melo Regis ${ }^{1}$, Ticiane Costa Farias ${ }^{1}$, Hédulla Karoliny de Souza \\ Lima Tavares ${ }^{1}$, Márlla Héllen do Nascimento Araújo ${ }^{1}$, Paula Frassinetti Oliveira \\ Cezário². \\ ${ }^{1}$ Universidade Federal de Campina Grande (UFCG). Email: \\ m.zilda melo@hotmail.com \\ ${ }^{2}$ Universidade Federal de Campina Grande (UFCG). Email: \\ paulafrassinetti22@gmail.com
}

\section{RESUMO}

INTRODUÇÃO: Os acidentes por animais peçonhentos configuram uma emergência clínica prevalente nas regiões tropicais e subtropicais, especialmente nas áreas rurais, e constituem, assim, um problema de saúde pública. Contudo, embora negligenciados, esses acidentes podem acarretar elevadas taxas de morbimortalidade. Esse tipo de envenenamento ocorre principalmente por picadas de escorpiões, serpentes, aranhas e abelhas. Ao analisar os dados relacionados aos acidentes, sugere-se que haja mais ações de vigilância em saúde, afim de facilitar as intervenções epidemiológicas de controle e prevenção. OBJETIVO: Identificar a taxa de detecção de acidentes por animais peçonhentos da cidade de Cajazeiras, Paraíba. MATERIAIS E METODOLOGIA: Trata-se de um estudo transversal de caráter descritivo e de natureza quantitativa. A população deste estudo categoriza-se por indivíduos acometidos por acidentes com animais peçonhentos nos anos de 2010 a 2015 na cidade de Cajazeiras, Paraíba. Os dados obtidos foram coletados do DATASUS pelo Sistema de Notificação de Agravos (SINAN), sendo descritos os valores absolutos. Os resultados foram agrupados em Classificação final, Evolução do caso, Sexo, Raça e Tipo de acidente, sempre combinados com Munícipio de notificação. As variáveis selecionadas foram "Ano acidente", "Município de notificação", "Classifica. Final", "Raça", "Sexo", "Tipo de acidente" e "Evolução do caso". Para análise dos dados utilizou-se o programa Microsoft Office Excel 2016. RESULTADOS E DISCUSSÕES: Em Cajazeiras-PB, houve 102 casos de acidentes com animais peçonhentos, sendo distribuídos entre os anos de 2010 a 2015, 81 acidentes foram classificados como de gravidade leve, 11 como de moderada, 5 como de grave e 5 foram ignorados. 91 casos evoluíram para a cura e 11 foram ignorados. 60 acidentes aconteceram com pacientes do sexo masculino e $42 \mathrm{com}$ pacientes do sexo feminino. Foram acometidos 83 indivíduos da raça parda, 9 da raça branca, 3 da raça preta e 7 foram ignorados. Acidentes ocasionados por serpentes atingiram o número de 43 casos, por aranha, 5 casos, por escorpião, 48 casos, por abelha, 2 casos e por outros, 2 casos. A média de casos por ano foi de 17. CONCLUSÕES: Analisando os resultados, notou-se que, entre os anos de 2010 a 2015, os casos de acidentes com animais peçonhentos concentraram-se especialmente nos originados por serpentes e escorpiões, cujo resultado se deve, provavelmente, à adaptação dessas espécies aos ambientes urbanos. Além disso, por ser um acidente de notificação compulsória sua ocorrência deve ser notificada pelos profissionais da saúde, a fim de auxiliar na implantação de programas e ações voltados para prevenção e combate dos acidentes por animais peçonhentos.

Palavras-chave: Animais peçonhentos; Taxa de detecção; Acidentes. 


\title{
A IMPORTÂNCIA DO CONHECIMENTO DA BIOMECÂNICA DO TRAUMA NOS FERIMENTOS POR ARMA DE FOGO
}

\author{
Luciano Mota Reis Neto ${ }^{1}$, David Sammuel Dantas Torres ${ }^{1}$, Yolanda de Melo \\ Omena Lira ${ }^{1}$, Wenya Cristiana de Almeida Abreu ${ }^{1}$, Luciana Modesto de Brito ${ }^{2}$. \\ ${ }^{1}$ Faculdade Santa Maria (FSM). Email: sobcontrole@hotmail.com \\ ${ }^{2}$ Faculdade Santa Maria (FSM). Email: lucianamodesto@hotmail.com
}

\begin{abstract}
RESUMO
INTRODUÇÃO: O entendimento de alguns princípios se faz necessário para a compreensão e interpretação dos diversos perfis de ferimentos por arma de fogo. Através do conhecimento científico e da avaliação detalhada dos padrões das lesões encontradas na vítima, o socorrista poderá interpretar qual o tipo de energia foi transferida e quais condições são ou poderão se tornar potencialmente fatais. Com base nos achados e na fisiopatologia das lesões locais, 0 serviço de emergência definirá qual melhor conduta a ser executada, pois saber onde examinar e como avaliar é tão importante quanto saber o que fazer depois que as lesões forem encontradas. OBJETIVO: $O$ presente estudo teve o objetivo discutir a importância do conhecimento da biomecânica nos ferimentos por arma de fogo, fornecendo ao socorrista 0 entendimento acerca dos fatores que afetam o tamanho da área de lesão, os níveis de energia, a avaliação dos orifícios de entrada e saída e os efeitos regionais dos ferimentos penetrantes. METODOLOGIA: Nesse tipo de pesquisa exploratória, realizou-se uma revisão integrativa qualitativa observacional específica através do estudo de casos retrospectivos, tendo como base as literaturas e manuais norteadores para adoção de protocolos de atendimento dos principais serviços de emergências médicas no mundo. RESULTADOS E DISCUSSÕES: A partir da análise literária, evidenciou-se a importância e a neccesidade da detalhada avaliação das lesões na vítima de arma de fogo, através da interpretação fundamenteda na biomecânica do trauma. Percebeu-se também, que a identificação precoce e a compreensão adequada são fundamentais para a escolha do tratamento apropriado da lesão, contribuindo significativamente no êxito durante $\mathrm{o}$ atendimento e na sobrevida do paciente assistido. CONSIDERAÇÕES FINAIS: A interpretação dos princípios da biomecânica do trauma na avaliação do doente traumatizado é a chave para descobrir lesões que de outra maneira passariam despercebidas e não suspeitadas. Nos ferimentos por arma de fogo, as lesões que não forem suspeitadas, detectadas e tratadas, contribuirão para a morbidade e a mortalidade decorrente deste tipo específico de lesão. O conhecimento da biomecânica fornece uma base científica para a suspeição de lesões não evidenciadas, sendo fundamental para profissionais dos serviços de urgências e emergências, principalmente no âmbito pré-hospitalar.
\end{abstract}

Palavras-chave: Ferimento por arma de fogo; Biomecânica do trauma; Avaliação. 


\title{
SÍFILIS CONGÊNITA: UM DESAFIO PARA A SAÚDE PÚBLICA
}

\author{
Rayllane Santos Nunes ${ }^{1}$, Marlla Héllen do Nascimento Araújo ${ }^{1}$, Marcos Alan de \\ Sousa Barbosa ${ }^{1}$, Rebeca Karollyne Rolim Ribeiro ${ }^{1}$, Rayanne de Sousa \\ Barbosa $^{2}$. \\ ${ }^{1}$ Universidade Federal de Campina Grande (UFCG). Email: \\ rayllanesnunes@gmail.com
}

${ }^{2}$ Faculdade Vale do Salgado (FVS). Email: anny-rayanne@hotmail.com

\begin{abstract}
RESUMO
INTRODUÇÃO: A sífilis congênita (SC) é o resultado da disseminação hematogênica pelo Treponema pallidum na gestante não tratada ou inadequadamente tratada para o seu concepto por via transplacentária ou por meio do contato da criança com o canal vaginal, se houver lesões genitais maternas. Apesar dos esforços para prevenção e controle, o número de casos registrados no Brasil continua a crescer. Dessa forma, surge a seguinte indagação: "Quais os fatores contribuem para a dificuldade no controle da SC?". A escolha do tema deu-se devido ao aumento na incidência dos casos no Brasil a cada ano. Nesse sentido, esse estudo tem como propósito contribuir com informações inerentes ao difícil controle da SC, servindo como fonte de novas pesquisas e permitindo maior aprofundamento científico. OBJETIVO: Identificar os principais desafios para a saúde pública relacionada ao controle da SC. METODOLOGIA: A pesquisa consiste em uma revisão bibliográfica realizada a partir de referências atualizadas, nacionais e internacionais, obtidas através das bases de dados online LILACS e MEDLINE. Foram selecionadas pesquisas publicadas entre os anos de 2011 e 2016, nos idiomas português e inglês e que contivessem em seus títulos ou resumos os seguintes descritores: Sífilis. Congênita. Saúde pública. Dos 27 artigos encontrados, sete foram selecionados para análise de acordo com os critérios de inclusão estabelecidos previamente. RESULTADOS E DISCUSSÃO: $\mathrm{Na}$ análise dos estudos, observa-se que, mesmo diante de todas as ações já desenvolvidas pelo Ministério da Saúde, fatores de caráter sociais e assistenciais contribuem para que a SC continue em vigência. Dentre os principais desafios a para a saúde pública destacam-se a subnotificação de sífilis na gestação em razão da realização do pré-natal de forma incompleta ou inadequada ou a não realização; a infrequência de parceiros no tratamento, além de aspectos socioeconômicos determinantes como a baixa escolaridade materna e a baixa renda, o que dificulta o acesso de gestantes a Unidades Básicas de Saúde e a laboratórios para realização de exames como o VDRL, o teste sorológico de triagem para detecção da sífilis na gestante, que deve ser realizado no primeiro e no terceiro trimestres da gestação. CONCLUSÃO: Embora a sífilis seja uma condição patológica cujas informações já estejam consideravelmente estabelecidas e acessíveis e o diagnóstico e tratamento sejam de baixo custo, são necessárias intervenções efetivas, com ênfase nos mecanismos de determinação social na SC de modo a guiar para prevenção do agravo e controle da doença.
\end{abstract}

Palavras-chave: Sífilis congênita; Saúde pública; Desafios. 


\title{
RECRUTAMENTO PULMONAR COM PRESSÃO POSITIVA EXPIRATÓRIA FINAL (PEEP) NA SÍNDROME DO DESCONFORTO RESPIRATÓRIO AGUDO (SDRA): UMA REVISÃO BIBLIOGRÁFICA
}

\author{
Francisco Jaime de Araujo Filho ${ }^{1}$, Lucas Gomes de Freitas Lima ${ }^{1}$, Samylly \\ Teixeira de Araújo ${ }^{1}$, Leonardo Pereira Tavares ${ }^{1}$, José Benício Dantas Neto ${ }^{2}$. \\ ${ }^{1}$ Universidade Federal Do Cariri (UFCA). Email: jaimetexera@hotmail.com \\ ${ }^{2}$ Faculdade Santa Maria (FSM).Email: j.beniciodantas@hotmail.com
}

\begin{abstract}
RESUMO
INTRODUÇÃO: As técnicas de mecânica ventilatória são imprescindíveis para estabilização de pacientes em péssimas condições respiratórias. Tais procedimentos são utilizados na SDRA, um dos principais grupos de doenças que acometem o sistema respiratório. Entretanto, complicações ocasionadas pelo uso crônico e, muitas vezes, desnecessário, dessas técnicas são registradas na literatura. Essa conjuntura demonstra a grande importância funcional que essas técnicas exercem sobre o tratamento emergencial de pacientes com agravos respiratórios de enorme magnitude. OBJETIVO: Analisar os benefícios e potenciais agravos advindos do uso das técnicas de recrutamento pulmonar com PEEP titulada ou baixa (parâmetros meramente fisiológicos). METODOLOGIA: Realizou-se busca na base de dados SciELO, utilizando os descritores "Mecânica Respiratória" (DeCS), "Tratamento SDRA" (DeCS) e "Pressão Expiratória Positiva Final" (DeCS). Foram rastreados textos em português e em inglês, publicados entre 1998 e agosto de 2017, dos quais apenas um atendeu às necessidades da revisão. Excluíram-se sete artigos por não agregarem o conhecimento específico. O artigo publicado no The Journal of the American Medical Association fora selecionado através dos descritores "Acute Respiratory Distress Syndrome" (DeCS) e "Positive End-Expiratory Pressure" (DeCS), os quais apresentaram outros 106 artigos em língua inglesa, que não atenderam ao objetivo proposto. No PUBMED, em pesquisa subsequente utilizando os descritores "Compared Peep Titration" e "ARDS", com o conector "and" e filtros "free full text", "5 years" e "humans" encontrou-se 4 artigos, dos quais um adequou-se ao objetivo. RESULTADOS E DISCUSSÕES: Um amplo estudo comparou o uso de diferentes níveis de PEEP e demonstrou que, aos 28 dias, o grupo experimental (PEEP titulada) apresentou maior mortalidade do que o grupo de controle (PEEP baixa). Tal pesquisa evidenciou, também, maior potencial de causar complicações em técnicas com PEEP titulado. Em contraposição, um estudo utilizado pelo Consenso Brasileiro de Ventilação Mecânica afirmou que elevar a PEEP na SDRA resulta em proteção contra lesão pulmonar associada à abertura e fechamento cíclicos de unidades alveolares recrutáveis. A partir disso, tal consenso estabelece parâmetros tendenciosamente elevados de PEEP que resultaram em mortalidade aos 28 dias quase duas vezes menor que PEEP de parâmetros menores. Somado a isso, outro artigo abordou a relação entre a PEEP baixa e a Pressão Transpulmonar(ptp), correlacionando-os com o alto risco de colapso alveolar na SDRA. Contudo, uma abundante variação de PEEP foi notada. É perceptível, assim, a grande discrepância entre valores de PEEP adequados. As situações averiguadas confirmam que não há um perfil concreto sobre o melhor tratamento no que diz respeito ao recrutamento pulmonar. Ademais, em quase 20 anos houve pouca evolução de pesquisas e, consequentemente, do conhecimento na área. CONCLUSÃO: Por conseguinte, os dados levantados e as discussões suscitadas demonstram a existência de incitante contrariedade quando se discute a melhor técnica para casos de SDRA. Diante disso, evidencia-se a necessidade de estudos inovadores e com maior capacidade de delimitar a área onde cada técnica é mais coerente, diminuindo, dessa maneira, o potencial risco que os pacientes são submetidos quando fazem uso de tais técnicas.
\end{abstract}

Palavras-chave: Síndrome do Desconforto Respiratório do Adulto; Respiração por Pressão Positiva Intrínseca; Respiração Artificial. 


\title{
PERFIL EPIDEMIOLÓGICO DA HANSENÍASE EM UMA CIDADE DA MESORREGIÃO DO SERTÃO PARAIBANO: UM ESTUDO ECOLÓGICO
}

\author{
Marcos Alan Sousa Barbosa ${ }^{1}$, Ana Paula Barbosa Nóbrega1, João Pedro \\ Maciel Capistrano ${ }^{1}$, Rayllane Santos Nunes ${ }^{1}$, Natália Bitu Pinto ${ }^{2}$. \\ ${ }^{1}$ Universidade Federal de Campina Grande (UFCG). Email: \\ alanbarbbosa@gmail.com \\ ${ }^{2}$ Universidade Federal de Campina Grande (UFCG). Email: \\ nataliabitu@gmail.com
}

\begin{abstract}
RESUMO
A hanseníase é uma doença infectocontagiosa causada pelo Mycobacterium leprae que se manifesta por sinais e sintomas dermatoneurológicos. É considerada um problema de saúde pública no Brasil por deter taxas de detecção e prevalência acima dos parâmetros internacionais estabelecidos pela Organização Mundial da Saúde (OMS). Por sua vez, o município de Cajazeiras, localizado na mesorregião do sertão paraibano, foi identificado no maior clauster (área de concentração) de detecção de novos casos da doença na Paraíba. Assim, objetiva-se traçar o perfil epidemiológico da hanseníase no município de Cajazeiras-PB, a fim de nortear políticas públicas futuras e refletir sobre as vigentes. Para isso, esse estudo trata-se de uma pesquisa epidemiológica, do tipo ecológico e transversal, referente aos anos de 2005 a 2015. Os dados foram coletados no Sistema de Informação de Agravos de Notificação (SINAN) disponível no Departamento de Informática do SUS (DATASUS). As variáveis estudadas foram: número de novos casos, índices de detecção, sexo, idade, classe operacional, grau de incapacidades e modo de detecção. Foram identificados, dessa forma, 623 novos casos de hanseníase durante o período estudado, apresentando um coeficiente de detecção médio 97,00/100.000 hab., considerado como hiperendêmico pela OMS (acima de $40,00 / 100.000$ hab.). Todos os coeficientes anuais compreendidos no período do estudo mantiveram-se como hiperendêmicos, com exceção do ano de 2014 que, mesmo assim, ainda situa-se na faixa de alta endemicidade (20,00 a 39,99/100.000 hab.). Os registros mostraram que a população masculina e a faixa etária economicamente ativa, compreendida entre 20-34 anos, foram as mais afetadas. Os modos de detecção de novos casos predominantes foram através de encaminhamentos e demandas espontâneas. Houve predomínio da forma paucibacilar, com exceção dos anos de 2014 e 2015, e do grau zero de incapacidade física. Contudo, os dados apontam para a prevalência do diagnóstico tardio e passividade dos serviços de saúde, uma vez que houve predomínio de formas infectantes da doença nos últimos anos analisados e ocorrência de proporção significativa de indivíduos com incapacidades físicas já no diagnóstico. A ocorrência de muitos indivíduos diagnosticados através da demanda espontânea, evidenciado nesse estudo, demonstram ainda a eficácia dos meios de comunicação na divulgação e esclarecimentos sobre a doença e suas incapacidades, mas também implica na potencialização do diagnóstico tardio, uma vez que o paciente é propenso a procurar ajuda quando os sinais da doença já estão evidentes. Logo, tais fatores colaboram para a manutenção do coeficiente de detecção na faixa hiperendêmica, fazendo-se necessário que o município promova e intensifique a descentralização das ações de controle da doença e estimule a busca ativa de novos casos. Também é importante que sejam desenvolvidas ações de acompanhamento durante e após o tratamento, com o objetivo de prevenir incapacidades físicas, manter o controle dos contatos intradomiciliares e consolidar 0 sistema de vigilância sobre a doença.
\end{abstract}

Palavras-chave: Epidemiologia. Hanseníase. Mycobacterium leprae. 


\title{
CONDUTAS DE URGÊNCIA UTILIZADAS EM FRATURAS DO ANEL PÉLVICO
}

\author{
Edilberto Costa Souza ${ }^{1}$, Marília Millena Remígio da Costa ${ }^{1}$, Vanessa Lima \\ Gonçalves ${ }^{1}$, Francisco Anderson de Sá Carvalho ${ }^{1}$, Sheylla Nadjane Batista \\ Lacerda ${ }^{2}$. \\ ${ }^{1}$ Faculdade Santa Maria (FSM), edilberto bioquimico@hotmail.com \\ ${ }^{2}$ Faculdade Santa Maria (FSM), sheyllabatista@bol.com.br
}

\section{RESUMO}

INTRODUÇÃO: As fraturas de anel pélvico são decorrentes de traumas com grande carga de energia cinética que ocorrem comumente em acidentes automotivos. Essas lesões representam uma urgência médica de importante atenção devido aos riscos imediatos e tardios que comprometem a vida humana ou deixam seqüelas. Os óbitos por esse tipo de fratura podem ocorrer em três momentos distintos: de forma imediata, precoce e tardia; a principal causa de morte imediata é a alteração hemodinâmica por lesão do plexo venoso lombossacral. OBJETIVOS: demonstrar a apresentação clínica das fraturas pélvicas, assim como os sinais e sintomas associados e a ação mais satisfatória na averiguação e tratamento, a fim de evitar intercorrências durante o manejo inadequado e reduzir as taxas de mortalidade. MÉTODOS: foi realizada uma revisão bibliográfica de caráter descritivo e qualitativo, baseada em leituras exploratórias e seletivas de artigos referentes ao tema proposto, pesquisados nas bases de dados da SCIELO, PUBMED utilizando os descritores (fracture of the pelvic ring) AND (bleeding) AND (stabilization). Após utilizar os critérios de inclusão dos últimos 5 anos e de estudos envolvendo seres humanos, foram localizados 11 artigos, dos quais 05 foram selecionados como sendo relevantes a partir da leitura do resumo. Foram critérios de exclusão: estudos em cadáveres, técnicas de estabilização pélvica citadas em trabalhos isolados. RESULTADOS E DISCUSSÃO: Na literatura, o diagnóstico de fratura do anel pélvico é presuposto através de atributos do exame físico em pacientes poli traumatizados; comol instabilidade pélvica, equimose no quadril, sinal de Destot, e diástase de sínfise púbica. Em pacientes hemodinamicamente instáveis a estabilização pélvica mecânica é imediatamente realizada, e o exame inicial preconizado, é a radiografia panorâmica na incidência anteroposterior seguida pela tomografia computadorizada. Uma compreensão completa da anatomia, achados radiográficos e exame físico inicial pode alertar sobre a presença de instabilidade pélvica que requer tratamento emergente, com isso, encontramos na prática clínica delineamentos como: compressão anteroposterior, compressão lateral e cisalhamento vertical. Torna-se portanto, imprescindível conceber o mecanismo da lesão traumática com o propósito de relacionar as possíveis lesões nos órgãos pélvicos. Sensato é, depois da estabilização hemodinâmica do paciente, efetuar novas incidências radiográficas complementares; chamadas de incidências oblíquas. A conduta imediata é a estabilização da pelve. $\mathrm{Na}$ qual, $\mathrm{O}$ fixador externo é muito útil especialmente na fase aguda, adquirindo uma redução aceitável e uma estabilidade adequada nas lesões parcialmente instáveis a fim de conter a hemorragia e fazer o tamponamento. Nenhuma diferença foi observada entre pacientes tratados com fixação interna e fixação de parafuso iliossacral percutâneo quando comparados à fixação externa $O$ manejo da instabilidade hemorrágica ligada à interrupção do anel pélvico envolve uma seqüência de eventos terapêuticos, que é mais importante do que os próprios eventos. CONCLUSÃO: Segundo os artigos analisados, existe um seguimento de cuidados importante que deve ser levado em importância quando se suspeita de fratura do anel pélvico. É essencial que o médico socorrista tenha em mente o dever de estabilizar a pelve e controlar a hemorragia local a fim de evitar complicações do quadro e favorecer um seguimento adequado

Palavras-chave: Fratura do anel pélvico; Estabilização; Hemorragia. 


\title{
POUCA ADESÃO POPULACIONAL AOS PROGRAMAS DE SAÚDE DA ATENÇÃO BÁSICA: UM RELATO DE EXPERIÊNCIA NO BAIRRO ASA DO MUNICÍPIO DE CAJAZEIRAS-PB
}

\author{
Maíra Pacheco Fraga ${ }^{1}$, Leandro Carvalho de Souza ${ }^{1}$, Leandro Santana \\ Ferreira ${ }^{1}$, Wengna Neves Matias ${ }^{1}$, Ocilma Barros de Quental ${ }^{2}$. \\ ${ }^{1}$ Faculdade Santa Maria (FSM). Email: inhafraga@hotmail.com \\ ${ }^{2}$ Faculdade Santa Maria (FSM). Email: ocilmaquental2011@hotmail.com
}

\begin{abstract}
RESUMO
INTRODUÇÃO: Levando em consideração as revoluções que o campo da saúde no Brasil vem avançando, as universidades tem investido na formação de profissionais capacitados e conhecedores do trabalho em saúde pública com vista na Estratégia de Saúde na família, assim, nós estudantes de medicina fomos incorporados a uma comunidade na cidade de Cajazeiras/PB. OBJETIVO: Relatar a pouca adesão populacional aos programas de saúde da atenção básica do Bairro Asa do Município de Cajazeiras-PB. METODOLOGIA: Trata-se de um relato de experiência vivenciado no município de Cajazeiras (PB), na UBS Américo Estrela Cartaxo, onde na oportunidade tivemos contato com algumas famílias do bairro adscrito, pelo qual foi observado importantes determinantes do processo saúde doença, entre eles destacavam-se a pouca adesão populacional aos programas instituídos pelo ministério da saúde e implementados pela Unidade Básica de Saúde. Os estudantes puderam observar durante visitas domiciliares e fazendo correlação com fundamentações literárias pertinente sobre o assunto, no intuito de contribuir para uma reflexão das transformações ocorridas no modelo de atenção à saúde e a adesão nos principais programas da atenção básica. DISCUSSÃO: O bairro conta com uma unidade Básica de Saúde que oferece assistência a aproximadamente 1.500 famílias, sendo estimada uma população em torno de 6.000 pessoas, o território encontra-se dividido 08 microáreas. A Unidade localiza-se em uma área estratégica, sendo de fácil acesso a população e perto das principais vias de entrada na comunidade. Com base na cobertura populacional do Programa Saúde da Família e dos Programas estabelecidos pelo Ministério da Saúde voltados para saúde da mulher, criança e adolescentes, adultos e idosos e saúde mental, encontrou-se em evidência a pouca aderência populacional aos programas instituídos e implementados pela Unidade Básica de Saúde. Dentre os fatores responsáveis pela baixa adesão foram relatados: a da falta de informações, pouco vínculo comunidade/equipe no que diz respeito aos atendimentos e serviços prestados, falta de comprometimento de familiares e alguns profissionais. Com isso, independentemente do programa, percebemos que a dificuldades impostas pela população e profissionais são preponderantes no quesito pouca adesão populacional. CONCLUSÃO: Deste modo, é de fundamental importância as mudanças nas rotinas da UBS em questão no que diz respeito ao desenvolvimento de atividades que incentivem os usuários a procurar os serviços oferecidos, como também é de extrema importância a adoção de uma maior aplicabilidade e implementação dos programas de saúde para fortalecer o vínculo usuário/profissional e contribuir para a melhoria da qualidade de vida da população.
\end{abstract}

Palavras-chave: Atenção básica; Adesão; População. 


\title{
MONITORIZAÇÃO HEMODINÂMICA INVASIVA
}

\author{
Vanessa Lima Gonçalves ${ }^{1}$, Edilberto Costa Souza ${ }^{1}$, Leonardo Victor de Moraes \\ Galdino $^{1}$, Alana Cristina Alves Garcia ${ }^{1}$, Sheylla Nadjane Batista Lacerda ${ }^{2}$. \\ ${ }^{1}$ Faculdade Santa Maria (FSM), Email: vanessalimagonçalves@hotmail.com \\ ${ }^{2}$ Faculdade Santa Maria (FSM), Email: sheyllabatista@bol.com.br
}

\begin{abstract}
RESUMO
OBJETIVO: Verificar, através de uma revisão sistemática, o que descreve a literatura sobre a utilização da monitoração hemodinâmica invasiva no cuidado a pacientes instavelmente enfermos. METODOLOGIA: Utilizou-se o método de revisão sistemática da literatura, com o objetivo de sintetizar resultados obtidos sobre um tema determinado, de maneira ordenada e sistemática, para contribuir com o conhecimento do tema em questão. Foram realizadas buscas nas bases de dados SciELO, PubMed e LILACS, com as palavras-chave: Monitoring AND hemodynamic AND invasive no período de Janeiro a setembro de 2017. RESULTADOS: A estratégia de busca resultou em 36 artigos, destes, 9 tratavam de monitorização nãoinvasiva, ficando a amostra final composta por 04 artigos. Constatou-se que dos 04 artigos selecionados, 02 citam algum tipo de monitorização hemodinâmica invasiva e apresentam conclusão sobre a temática. Observa-se, no geral, que uma grande parte dos autores sente a necessidade de substituição dos métodos invasivos por outros minimamente invasivos aliandose aos métodos não invasivos, numa tentativa de sobrepor os benefícios sobre os riscos dos métodos invasivos. CONCLUSÕES: A monitorização hemodinâmica invasiva ainda tem extrema valia para o cuidado ao paciente enfermo e principalmente àquele em estado crítico, todavia tem-se notado uma busca crescente por métodos de avaliação não invasivas destes pacientes. Outrossim, vê-se também que os benefícios dos métodos invasivos estão diretamente relacionados à sua correta interpretação de resultados associado a um pleno conhecimento teórico/científico por parte dos profissionais responsáveis por instituir tais monitorizações. Ressalta-se que o interesse em substituir os métodos invasivos pelos minimamente invasivos e não invasivos, ainda traz grande dificuldade em determinar se esses métodos menos invasivos tem a mesma confiabilidade que os invasivos.
\end{abstract}

Palavras-chave: Monitorização; Hemodinâmica; Invasiva. 


\title{
FATORES QUE INTERFEREM NA HIPERBILIRRUBINEMIA NEONATAL: UMA REVISÃO DE LITERATURA
}

\author{
Dulcy Dávyla Freire Nascimento', Ana Maria Souza Costa Barros ${ }^{1}$, Nathalie \\ Ramos Formiga Rolim¹, Joyce Flávia da Silva Leal'1, Ana Emília Formiga \\ Marques². \\ ${ }^{1}$ Faculdade Santa Maria (FSM). Email: dulcyfn@hotmail.com \\ ${ }^{2}$ Faculdade de Medicina de Juazeiro do Norte (FMJ). Email: \\ anaemiliaformiga@hotmail.com
}

\begin{abstract}
RESUMO
INTRODUÇÃO: A icterícia é um dos problemas mais frequentes no período neonatal e corresponde a expressão clínica de hiperbilirrubinemia, que é definida como a concentração sérica de bilirrubina indireta $(\mathrm{BI})$ maior que 1,3 a $1,5 \mathrm{mg} / \mathrm{dl}$ ou de bilirrubina direta $(\mathrm{BD})$ superior a $1,5 \mathrm{mg} / \mathrm{dl}$, desde que esta represente mais do que $10 \%$ do valor de bilirrubina total (BT). OBJETIVOS: Realizar levantamento literário sobre os fatores que interferem na hiperbilirrubinemia neonatal. MATEIRAIS E MÉTODOS: Foram selecionados, por meio de busca eletrônica, artigos das bases de dados MEDLINE, LILACS e SciELO publicados de 2007 a 2017. Para o levantamento foram utilizados os descritores hiperbilirrubinemia e hiperbilirrubinemia neonatal. RESULTADOS E DISCUSSÕES: Os principais fatores elencados pelos autores foram: fatores epidemiológicos, populacionais, familiares e maternos interferem nos níveis de bilirrubina nos RN de forma consciente. Dentre os fatores neonatais, ressaltamse os aspectos relacionados à prática do aleitamento materno, em especial a perda de peso e o tempo de permanência hospitalar, que podem interferir de forma significativa na bilirrubinemia. A denominada icterícia pela falta de aleitamento materno na 1 semana de vida caracteriza-se por acompanhar-se de perda de peso no 3 dia de vida em relação ao peso de nascimento maior que $5 \%$ nos neonatos em aleitamento materno. A explicação mais favorável para a sua ocorrência é a ingestão inadequada de leite acarretando aumento da circulação êntero-hepática de bilirrubina. A icterícia pela falta de aleitamento materno também tem sido associada nos últimos anos à alta hospitalar antes de 48 horas de vida, uma vez que existe uma tendência de encurtar o tempo de internação hospitalar com a finalidade de diminuir os custos hospitalares. A maior parte dos $\mathrm{RN}$ reinternados após a saída da maternidade está em aleitamento materno exclusivo, sendo problemas com a oferta láctea e a desidratação a principal causa de hiperbilirrubinemia. CONCLUSÕES: A partir desses estudos percebe-se que fator nutricional, principalmente a falta do aleitamento materno interfere significativamente na hiperbilirrubinemia. Destaca-se a importância de um maior acompanhamento nas primeiras 48 horas de vida e do acompanhamento multiprofissional quanto ao aleitamento materno.
\end{abstract}

Palavras-chaves: Hiperbilirrubinemia neonatal; Pediatria; Icterícia neonatal. 